PROCEEDINGS OF THE

AMERICAN MATHEMATICAL SOCIETY

Volume 135, Number 7, July 2007, Pages 2141-2149

S 0002-9939(07)08813-2

Article electronically published on March 2, 2007

\title{
UNIQUE CONTINUATION FOR THE SCHRÖDINGER EQUATION WITH GRADIENT VECTOR POTENTIALS
}

\author{
HONGJIE DONG AND WOLFGANG STAUBACH
}

(Communicated by David S. Tartakoff)

\begin{abstract}
We obtain unique continuation results for Schrödinger equations with time dependent gradient vector potentials. This result with an appropriate modification also yields unique continuation properties for solutions of certain nonlinear Schrödinger equations.
\end{abstract}

\section{INTRODUCTION}

In this paper we study unique continuation properties of solutions to the Schrödinger equations having time dependent gradient magnetic vector potentials. Specifically, we consider equations of the form

$$
\begin{aligned}
& i \partial_{t} u+\Delta_{x} u=p_{1}(x, t) \cdot \nabla_{x} u+p_{2}(x, t) \cdot \nabla_{x} \bar{u} \\
& \quad+V_{1}(x, t) u+V_{2}(x, t) \bar{u} \quad \text { on } \mathbb{R}^{d} \times(0,1),
\end{aligned}
$$

where the vector potentials $p_{1}$ and $p_{2}$ and the scalar potentials $V_{1}, V_{2}$ are assumed to belong to certain Banach spaces considered in the work of Ionescu and Kenig [6]. The unique continuation results for solutions to equation (1.1) can be used to obtain unique continuation properties of solutions to nonlinear Schrödinger equations of type

$$
i \partial_{t} u+\Delta_{x} u=P\left(u, \bar{u}, \nabla_{x} u, \nabla_{x} \bar{u}\right) \text { on } \mathbb{R}^{d} \times(0,1),
$$

where $P: \mathbb{C} \times \mathbb{C} \times \mathbb{C}^{d} \times \mathbb{C}^{d} \rightarrow \mathbb{C}$ is a polynomial.

Unique continuation for the solutions of Schrödinger equations has been studied by various authors e.g. Bourgain [1, Kenig and Ionescu [5], 6], Kenig, Ponce and Vega [7, and Escauriaza, Kenig, Ponce and Vega [2].

The main goal here is to find sufficient conditions on the solution $u$ of the linear equation (1.1), at two different times $t=0$ and $t=1$, which guarantee that $u \equiv 0$. In the case of nonlinear Schrödinger equation (1.2) the uniqueness result is obtained from the information on the difference of two possible solutions at two different times.

Our approach follows that of [2, but since we also have to deal with the presence of the gradient vector potential, we need some estimates in the spirit of [ 6 .

Received by the editors March 18, 2006.

2000 Mathematics Subject Classification. Primary 35B37.

Key words and phrases. Carleman inequalities; uniqueness of solutions.

(C)2007 American Mathematical Society Reverts to public domain 28 years from publication 
Let $H=i \partial_{t}+\Delta$ denote the Schrödinger operator and denote by $H^{1}$ the Sobolev space of tempered distributions $u$ for which $(1-\Delta)^{\frac{1}{2}} u \in L^{2}\left(\mathbb{R}^{d}\right)$. For any $k \in \mathbb{Z}^{d}$ let $Q_{k}$ denote the cube

$$
\left\{x \in \mathbb{R}^{d} ; x_{j} \in\left[k_{j}-\frac{1}{2}, k_{j}+\frac{1}{2}\right), j=1, \ldots, d\right\} .
$$

Following $[6]$, for $p, q \in[1, \infty]$ we define the Banach space $B^{p, q}\left(\mathbb{R}^{d}\right)$ using the norm

$$
\|f\|_{B^{p, q}}:=\left\{\sum_{k \in \mathbb{Z}^{d}}\|f\|_{L^{q}\left(Q_{k}\right)}^{p}\right\}^{\frac{1}{p}}, 1 \leq p<\infty,\|f\|_{B^{\infty, q}}:=\sup _{k \in \mathbb{Z}^{d}}\|f\|_{L^{q}\left(Q_{k}\right)} .
$$

From the definition it follows that $B^{p, p}=L^{p}, 1 \leq p \leq \infty$, and

$$
B^{p_{1}, q_{1}} \hookrightarrow B^{p_{2}, q_{2}} \text { if } q_{1} \geq q_{2} \text { and } p_{1} \leq p_{2} .
$$

Our main result for the linear Schrödinger equation is the following:

Theorem 1.1. Let $u \in C\left([0,1]: H^{1}\right)$ be a solution of the equation

$$
H u=V_{1} u+V_{2} \bar{u}+p_{1} \cdot \nabla_{x} u+p_{2} \cdot \nabla_{x} \bar{u} \quad \text { on } \mathbb{R}^{d} \times(0,1),
$$

where

$$
V_{1}, V_{2} \in B_{x}^{2, \infty} L_{t}^{\infty}\left(\mathbb{R}^{d} \times[0,1]\right), \quad\left|p_{1}\right|,\left|p_{2}\right| \in B_{x}^{1, \infty} L_{t}^{\infty}\left(\mathbb{R}^{d} \times[0,1]\right) .
$$

If there exists $a \geq c_{0}\left(d,\left\|p_{1}\right\|_{L_{t, x}^{\infty}},\left\|p_{2}\right\|_{L_{t, x}^{\infty}}\right)$ such that

$$
u(\cdot, 0), u(\cdot, 1) \in H^{1}\left(e^{a|x|^{2}}\right),
$$

then $u \equiv 0$.

Remark 1.2. In some sense, this theorem is a generalization of the result obtained in [2], to the case of Schrödinger operators with gradient vector potentials. To satisfy (1.4), we need $p_{j}, V_{j}, j=1,2$, to be bounded and decay with certain rates at infinity. For example, all Schwartz functions and all functions of the form $1 /\left(1+|x|^{d+\alpha}\right), \alpha>$ 0 , satisfy this assumption. By going through the proof of Theorem 1.1 and using the Sobolev embedding theorem, one can see that the condition of $V_{j}$ 's in (1.4) can be relaxed to

$$
\begin{gathered}
V_{1}, V_{2} \in\left(B_{x}^{2, \infty}+B_{x}^{\frac{2 d}{d+1}, 2 d}\right) L_{t}^{\infty}\left(\mathbb{R}^{d} \times[0,1]\right) \quad \text { if } d \geq 2, \\
V_{1}, V_{2} \in\left(B_{x}^{2, \infty}+B_{x}^{\frac{1}{1-\gamma}, \frac{2}{1-2 \gamma}}\right) L_{t}^{\infty}\left(\mathbb{R}^{d} \times[0,1]\right) \quad \text { if } d=1,
\end{gathered}
$$

for any $\gamma \in(0,1 / 2]$. However, at present we do not know if (1.4) can be improved significantly.

We show that for the nonlinear Schrödinger equation, one has the following unique continuation property.

Theorem 1.3. Consider

$$
H u=P\left(u, \bar{u}, \nabla_{x} u, \nabla_{x} \bar{u}\right) \quad \text { on } \mathbb{R}^{d} \times(0,1),
$$

and let $X$ be either $L^{2}\left(\mathbb{R}^{d}\right)$ or $B^{1,2}\left(\mathbb{R}^{d}\right)$ and $J^{s}:=\left(1-\Delta_{x}\right)^{\frac{s}{2}}$. Assume that $u_{1}, u_{2}$ are solutions to (1.6) and we have

$$
\begin{gathered}
J^{s} u_{1}, J^{s} u_{2} \in C([0,1]: X), s>d / 2+3, \\
\left\|J^{s} u_{1}\right\|_{C([0,1]: X)} \leq N, \quad\left\|J^{s} u_{2}\right\|_{C([0,1]: X)} \leq N, \\
\left|P\left(z_{1}, z_{2}, w_{1}, w_{2}\right)\right| \leq N\left(\left|z_{1}\right|^{2}+\left|z_{2}\right|^{2}+\left|w_{1}\right|^{2}+\left|w_{2}\right|^{2}\right),
\end{gathered}
$$


for a constant $N>0$ and any $\left|\left(z_{1}, z_{2}, w_{1}, w_{2}\right)\right| \leq 1$. If $u=u_{1}-u_{2}$ satisfies (1.5) for some $a \geq c_{0}(d, N)$, then $u_{1} \equiv u_{2}$ in $\mathbb{R}^{d} \times[0,1]$.

Remark 1.4. The result of Theorem 1.3 can be extended to more general nonlinearities of the form $F\left(x, t, u, \bar{u}, \nabla_{x} u, \nabla_{x} \bar{u}\right)$, where one only needs to assume that

$$
\begin{aligned}
& F\left(x, t, u_{1}, \bar{u}_{1}, \nabla_{x} u_{1}, \nabla_{x} \bar{u}_{1}\right)-F\left(x, t, u_{1}, \bar{u}_{1}, \nabla_{x} u_{1}, \nabla_{x} \bar{u}_{1}\right) \\
& =p_{1}(x, t) \cdot \nabla_{x} u+p_{2}(x, t) \cdot \bar{\nabla}_{x} u+V_{1}(x, t) u+V_{2}(x, t) \bar{u}
\end{aligned}
$$

with $u=u_{1}-u_{2}$, for some $V_{1}, V_{2}, p_{1}, p_{2}$ as in (1.4) (See Kenig et al. [6] and [8].)

The rest of the article is organized as follows: In section 2 we shall prove suitable Carleman estimates which are the main tools in obtaining unique continuation results. In section 3 we shall establish lower bounds for the $L^{2}$ space-time norm of the solutions to (1.1) and (1.2). These lower bounds combined with the Carleman estimates of section 2 yield the desired unique continuation properties.

\section{Carleman estimates And upper bounds}

Let $\phi$ be a smooth function on $\mathbb{R}$ with the following properties: $\phi(0)=0, \phi^{\prime}$ nonincreasing, $\phi^{\prime}(r)=1$ if $r \leq 1$ and $\phi^{\prime}(r)=0$ if $r \geq 2$. For any $\lambda \geq 1$, let $\phi_{\lambda}(r)=\lambda \phi(r / \lambda)$. As in [6], for any $\beta>0, \lambda>0$, we define the Banach space $X=X_{\lambda}\left(\mathbb{R}^{d} \times \mathbb{R}\right)$ using the norm

$$
\|f\|_{X}:=\inf _{f_{1}+f_{2}=f}\left[\left\|f_{1}\right\|_{B_{x}^{1,2} L_{t}^{2}}+\lambda^{3}\left\|J^{1} f_{2}\right\|_{L_{t}^{1} L_{x}^{2}}\right] .
$$

We also define the Banach Space $X^{\prime}=X_{\beta, \lambda}^{\prime}\left(\mathbb{R}^{d} \times \mathbb{R}\right)$ using the norm

$$
\|u\|_{X^{\prime}}:=\max \left\{\left\|J^{1 / 2} u\right\|_{L_{t}^{\infty} L_{x}^{2}},\left\|J^{1} u\right\|_{B_{x}^{\infty, 2} L_{t}^{2}},\left\|\left(1+\beta \phi_{\lambda}^{\prime}\left(x_{1}\right)\right) u\right\|_{B_{x}^{\infty, 2} L_{t}^{2}}\right\} .
$$

We will need the following Carleman-type estimate:

Lemma 2.1. There are constants $\bar{C}, C_{0}$ and $m$ depending only on $d$ such that

$$
\left\|e^{\beta \phi_{\lambda}\left(x_{1}\right)} u\right\|_{L_{x}^{2} L_{t}^{2}}+\left\|e^{\beta \phi_{\lambda}\left(x_{1}\right)}|\nabla u|\right\|_{B_{x}^{\infty, 2} L_{t}^{2}} \leq \bar{C}\left\|e^{\beta \phi_{\lambda}\left(x_{1}\right)} H u\right\|_{X}
$$

for any $u \in C\left(\mathbb{R}: H^{1}\right)$ with $u(\cdot, t) \equiv 0$ for $t \notin[0,1]$, any $\beta \in[1, \infty)$ and any $\lambda \geq C_{0} \beta^{m}$.

Proof. In [6], Ionescu and Kenig established the Carleman estimate

$$
\left\|e^{\beta \phi_{\lambda}\left(x_{1}\right)} u\right\|_{X^{\prime}} \leq \bar{C}\left\|e^{\beta \phi_{\lambda}\left(x_{1}\right)} H u\right\|_{X},
$$

under the same assumptions as in Lemma 2.1. Using the $B^{\infty, 2}$ boundedness of pseudodifferential operators of order zero obtained in [4, one can show that

$$
\begin{gathered}
\left\|e^{\beta \phi_{\lambda}\left(x_{1}\right)} u\right\|_{L_{x}^{2} L_{t}^{2}}+\left\|e^{\beta \phi_{\lambda}\left(x_{1}\right)}|\nabla u|\right\|_{B_{x}^{\infty, 2} L_{t}^{2}} \leq C \max \left\{\left\|J^{1 / 2}\left(e^{\beta \phi_{\lambda}\left(x_{1}\right)} u\right)\right\|_{L_{t}^{\infty} L_{x}^{2}},\right. \\
\left.\left\|J^{1}\left(e^{\beta \phi_{\lambda}\left(x_{1}\right)} u\right)\right\|_{B_{x}^{\infty, 2} L_{t}^{2}},\left\|\left(1+\beta \phi_{\lambda}^{\prime}\left(x_{1}\right)\right) e^{\beta \phi_{\lambda}\left(x_{1}\right)} u\right\|_{B_{x}^{\infty, 2} L_{t}^{2}}\right\}
\end{gathered}
$$

for some constant $C$.

Without loss of generality, in the sequel we assume that $m \geq 2$. Lemma 2.1 implies the following theorem. 
Theorem 2.2. There exist constants $C, C_{0}, m \geq 2$ depending only on $d$ such that

$$
\begin{gathered}
\left\|e^{\beta \phi_{\lambda}(|x| / \sqrt{d})} v\right\|_{L_{x}^{2} L_{t}^{2}}+\left\|e^{\beta \phi_{\lambda}(|x| / \sqrt{d})}|\nabla v|\right\|_{B_{x}^{\infty, 2} L_{t}^{2}} \\
\leq C\left\|e^{\beta \phi_{\lambda}(|x|)} H v\right\|_{X}+C \lambda^{3} \sum_{j=0}^{1}\left\|J^{1}\left(e^{\beta \phi_{\lambda}(|x|)} v(x, j)\right)\right\|_{L_{x}^{2}}
\end{gathered}
$$

for any $v \in C\left(\mathbb{R}: H^{1}\right), \beta \in[1, \infty)$ and any $\lambda \geq C_{0} \beta^{m}$.

Proof. First we claim that there exist constants $C, m \geq 2$ depending only on $d$ such that

$$
\begin{gathered}
\left\|e^{\beta \phi_{\lambda}\left(x_{1}\right)} v\right\|_{L_{x}^{2} L_{t}^{2}}+\left\|e^{\beta \phi_{\lambda}\left(x_{1}\right)} \mid \nabla v\right\|_{B_{x}^{\infty, 2} L_{t}^{2}} \\
\leq C\left\|e^{\beta \phi_{\lambda}\left(x_{1}\right)} H v\right\|_{X}+C \lambda^{3} \sum_{j=0}^{1}\left\|J^{1}\left(e^{\beta \phi_{\lambda}\left(x_{1}\right)} v(x, j)\right)\right\|_{L_{x}^{2}}
\end{gathered}
$$

for any $v \in C\left(\mathbb{R}: H^{1}\right), \beta \in[1, \infty)$ and any $\lambda \geq C_{0} \beta^{m}$.

To prove (2.2), for any $\varepsilon \in(0,1 / 10)$ we define a smooth cutoff function $\psi_{\varepsilon}: \mathbb{R} \rightarrow$ $[0,1]$ such that

$$
\begin{cases}\psi_{\varepsilon} \equiv 1, & \text { on }[2 \varepsilon, 1-2 \varepsilon] \\ \psi_{\varepsilon} \equiv 0, & \text { on }(-\infty, \varepsilon] \cup[1-\varepsilon, \infty)\end{cases}
$$

and $\psi_{\varepsilon}$ is increasing on $(-\infty, 1 / 2)$ and decreasing on $(1 / 2, \infty)$. Since $u(x, t)=$ $v(x, t) \psi_{\varepsilon}(t)$ satisfies the condition in Lemma 2.1 we have

$$
\begin{gathered}
\left\|e^{\beta \phi_{\lambda}\left(x_{1}\right)} v \psi_{\varepsilon}\right\|_{L_{x}^{2} L_{t}^{2}}+\left\|e^{\beta \phi_{\lambda}\left(x_{1}\right)}\left|\psi_{\varepsilon} \nabla v\right|\right\|_{B_{x}^{\infty, 2} L_{t}^{2}} \\
\leq C\left\|e^{\beta \phi_{\lambda}\left(x_{1}\right)} H v\right\|_{X}+C \lambda^{3}\left\|J^{1}\left(e^{\beta \phi_{\lambda}\left(x_{1}\right)} \psi_{\varepsilon}^{\prime} v(x, t)\right)\right\|_{L_{t}^{1} L_{x}^{2}} .
\end{gathered}
$$

Letting $\varepsilon \rightarrow 0$ yields (2.2) immediately. Notice that this estimate still holds with $x_{j}$ or $-x_{j}$ in place of $x_{1}$, where $j=1,2, \cdots, d$. Thus to prove (2.2) it suffices to use the monotonicity of $\phi_{\lambda}$, the obvious inequalities

$$
|x| / \sqrt{d} \leq \max _{j}\left|x_{j}\right|, \quad e^{\beta \phi_{\lambda}(|x| / \sqrt{d})} \leq \sum_{j=1}^{d}\left(e^{\beta \phi_{\lambda}\left(x_{j}\right)}+e^{\beta \phi_{\lambda}\left(-x_{j}\right)}\right)
$$

and the triangle inequality. This finishes the proof of the theorem.

We define the operator

$$
H_{V, p} u=H u-V_{1} u-V_{2} \bar{u}-p_{1} \cdot \nabla u-p_{2} \cdot \nabla \bar{u} .
$$

Corollary 2.3. Under the assumption of Theorem 2.2 and the additional assumptions

$$
\left\|\left|p_{1}\right|\right\|_{B_{x}^{1, \infty} L_{t}^{\infty}}+\left\|\left|p_{2}\right|\right\|_{B_{x}^{1, \infty} L_{t}^{\infty}} \leq \frac{1}{2 C}, \quad\left\|V_{1}\right\|_{B_{x}^{2, \infty} L_{t}^{\infty}}+\left\|V_{2}\right\|_{B_{x}^{2, \infty} L_{t}^{\infty}} \leq \frac{1}{2 C},
$$

with $C$ as in Theorem 1.1, we have

$$
\begin{gathered}
\left\|e^{\beta \phi_{\lambda}(|x| / \sqrt{d})} v\right\|_{L_{x}^{2} L_{t}^{2}}+\left\|e^{\beta \phi_{\lambda}(|x| / \sqrt{d})}|\nabla v|\right\|_{B_{x}^{\infty, 2} L_{t}^{2}} \\
\leq N\left\|e^{\beta \phi_{\lambda}(|x|)} H_{V, p} v\right\|_{X}+N \lambda^{3} \sum_{j=0}^{1}\left\|J^{1}\left(e^{\beta \phi_{\lambda}(|x|)} v(x, j)\right)\right\|_{L_{x}^{2}},
\end{gathered}
$$

with $N=2 C$. 
Proof. This follows by applying the inequality (2.2) to $H v=H_{V, p} v+V_{1} v+V_{2} \bar{v}+p_{1}$. $\nabla v+p_{2} \cdot \nabla \bar{v}$ and observing that the definition of the space $X$, the Hölder inequality and the assumptions (2.4) imply

$$
\begin{aligned}
& C\left\|e^{\beta \phi_{\lambda}\left(x_{1}\right)}\left(V_{1} v+V_{2} \bar{v}+p_{1} \cdot \nabla v+p_{2} \cdot \nabla \bar{v}\right)\right\|_{X} \\
\leq & \frac{1}{2}\left(\left\|e^{\beta \phi_{\lambda}\left(x_{1}\right)} v\right\|_{L_{x}^{2} L_{t}^{2}}+\left\|e^{\beta \phi_{\lambda}\left(x_{1}\right)}|\nabla v|\right\|_{B_{x}^{\infty, 2} L_{t}^{2}}\right) .
\end{aligned}
$$

The result of the corollary now follows by using (2.3).

We are now ready to state and prove the main theorem of this section.

Theorem 2.4. Let $u \in C\left([0,1]: H^{1}\right)$ be a solution of (1.3) and assume that for some $a>0$ one has

$$
u(\cdot, 0), u(\cdot, 1) \in H^{1}\left(e^{a|x|^{2}}\right), \quad \sum_{j=0}^{1}\|u(x, j)\|_{H^{1}\left(e^{a|x|^{2}}\right)} \leq K,
$$

for some constant $K>0$. Then there exist $N=N(d)>0$ and $R_{0}>0$ depending only on $d, a \wedge 1, K, p_{1}, p_{2}, V_{1}, V_{2}$, the $L^{2}\left([0,1]: H^{1}\right)$ norm of $u$, such that for all $R \geq R_{0}$

$$
\|u\|_{L^{2}(\{R \leq|x| \leq R+1\} \times[0,1])}+\||\nabla u|\|_{L^{2}(\{R \leq|x| \leq R+1\} \times[0,1])} \leq N e^{-a \frac{R^{2}}{36 d}} .
$$

Proof. We choose $R_{0} \geq 1$ sufficiently large such that the corresponding norms in $\left\{x:|x| \geq R_{0}\right\} \times[0,1]$ of $V_{1}, V_{2}, p_{1}, p_{2}$ are smaller than $1 /(4 C)$, where $C$ is the constant in Theorem 1.1. Let $\mu: \mathbb{R}^{d} \rightarrow[0,1]$ be a smooth function such that $\mu \equiv 0$ for $|x| \leq 1$ and $\mu \equiv 1$ for $|x| \geq 2$. For any $R>0$, we set

$$
\begin{gathered}
\mu_{R}(x)=\mu(x / R), \quad u_{R}=u \mu_{R}, \\
V_{R, j}=V_{j} \chi_{|x| \geq R}, \quad p_{R, j}=p_{j} \chi_{|x| \geq R}, j=1,2 .
\end{gathered}
$$

A direct computation shows that $H_{V_{R}, p_{R}} u_{R}=e_{R}$, where

$$
e_{R}=-\nabla \mu_{R} \cdot p_{1} u-\nabla \mu_{R} \cdot p_{2} \bar{u}+\Delta \mu_{R} u+2 \nabla \mu_{R} \cdot \nabla u \text {. }
$$

We set $\beta=a R / 3, \lambda=C_{0} \beta^{m}$, and use Corollary 2.3 with $u_{R}, V_{R}, p_{R}$ in place of $v, V$ and $p$ and obtain

$$
\left\|e^{\beta \phi_{\lambda}(|x| / \sqrt{d})} u_{R}\right\|_{L_{x}^{2} L_{t}^{2}}+\left\|e^{\beta \phi_{\lambda}(|x| / \sqrt{d})}\left|\nabla u_{R}\right|\right\|_{B_{x}^{\infty, 2} L_{t}^{2}} \leq N\left(I_{1}+I_{2}\right),
$$

where

$$
I_{1}=\left\|e^{\beta \phi_{\lambda}(|x|)} e_{R}\right\|_{X}, \quad I_{2}=\lambda^{3} \sum_{j=0}^{1}\left\|J^{1}\left(e^{\beta \phi_{\lambda}(|x|)} u_{R}(x, j)\right)\right\|_{L_{x}^{2}} .
$$

Estimate of $I_{2}$ : It is clear that

$$
I_{2} \leq \lambda^{3}(\beta+1)\left(\sum_{j=0}^{1}\left\|e^{\beta \phi_{\lambda}(|x|)} u_{R}(x, j)\right\|_{L_{x}^{2}}+\left\|e^{\beta \phi_{\lambda}(|x|)}\left|\nabla u_{R}(x, j)\right|\right\|_{L_{x}^{2}}\right) .
$$

On the set $\{x:|x| \geq R\}$, we have

$$
\beta \phi_{\lambda}(|x|) \leq a|x|^{2} / 3 \leq a|x|^{2}-2 a R^{2} / 3 .
$$

Therefore, for $R$ sufficiently large

$$
I_{2} \leq N(d) a^{m+1} R^{m+1} e^{-2 a R^{2} / 3} \sum_{j=0}^{1}\|u(x, j)\|_{H^{1}\left(e^{a|x|^{2}}\right)} \leq 1 .
$$


This gives the estimate of $I_{2}$.

Estimate of $I_{1}$ : Since $e_{R}$ is supported in the annulus $\{R \leq|x| \leq 2 R\}$, Hölder's inequality yields

$$
\begin{gathered}
I_{1} \leq e^{2 a R^{2} / 3}\left\|-\nabla \mu_{R} \cdot p_{1} u-\nabla \mu_{R} \cdot p_{2} \bar{u}+\Delta \mu_{R} u+2 \nabla \mu_{R} \cdot \nabla u\right\|_{B_{x}^{1,2} L_{t}^{2}} \\
\leq e^{2 a R^{2} / 3}\left[\|u\|_{L_{x}^{2} L_{t}^{2}}\left(\left\|\nabla \mu_{R} \mid\left(\left|p_{1}\right|+\left|p_{2}\right|\right)\right\|_{B_{x}^{2, \infty} L_{t}^{\infty}}+\left\|\Delta \mu_{R}\right\|_{B_{x}^{2, \infty}}\right)\right. \\
\left.\quad+2\|\nabla u\|_{L_{x}^{2} L_{t}^{2}}\left\|\nabla \mu_{R}\right\|_{B_{x}^{2, \infty}}\right] \\
\leq N(d) e^{2 a R^{2} / 3} R^{d / 2-1}\left(1+\left\|\left|p_{1}\right|\right\|_{L_{t, x}^{\infty}}+\left\|\left|p_{2}\right|\right\|_{L_{t, x}^{\infty}}\right)\|u\|_{H_{x}^{1} L_{t}^{2}} \leq e^{a R^{2}},
\end{gathered}
$$

for $R$ large enough. This completes the estimate of $I_{1}$, and using (2.5) we get for $R$ sufficiently large

$$
\left\|e^{\beta \phi_{\lambda}(|x| / \sqrt{d})} u_{R}\right\|_{L_{x}^{2} L_{t}^{2}}+\left\|e^{\beta \phi_{\lambda}(|x| / \sqrt{d})}\left|\nabla u_{R}\right|\right\|_{B_{x}^{\infty, 2} L_{t}^{2}} \leq N(d) e^{a R^{2}} .
$$

Notice that in the annulus $\{x ; 6 \sqrt{d} R \leq|x| \leq 6 \sqrt{d} R+1\}$, we have $u_{R}=u$ and $\beta \phi_{\lambda}(|x| / \sqrt{d}) \geq 2 a R^{2}$. From (2.6), we deduce

$$
e^{2 a R^{2}}\|u\|_{H^{1}(\{6 \sqrt{d} R \leq|x| \leq 6 \sqrt{d} R+1\}) L_{t}^{2}} \leq N(d) e^{a R^{2}} .
$$

Upon a change of variable, the theorem is proved.

\section{LOWER BOUNDS}

In this section, we shall give lower bounds for the $H^{1}$ norms of the solutions to the equation (1.3) in the annulus $\{R \leq|x| \leq R+1\} \times[0,1]$. We follow the line in section 3 of [2]. The following lemma is similar to Lemma 3.1 of [2].

Lemma 3.1. Assume that $R>0$ and $\varphi:[0,1] \rightarrow \mathbb{R}$ is a smooth function. Then there exist $c=c\left(d,\left\|\varphi^{\prime}\right\|_{L^{\infty}},\left\|\varphi^{\prime \prime}\right\|_{L^{\infty}}\right)>0, N=N(d)$ such that the estimate

$$
\begin{gathered}
\alpha^{3 / 2} R^{-2}\left\|\left|x R^{-1}+\varphi(t) e_{1}\right| e^{\alpha\left|x R^{-1}+\varphi(t) e_{1}\right|^{2}} v\right\|_{L_{x}^{2} L_{t}^{2}} \\
+\alpha^{1 / 2} R^{-1}\left\|e^{\alpha\left|x R^{-1}+\varphi(t) e_{1}\right|^{2}} \nabla v\right\|_{L_{x}^{2} L_{t}^{2}} \leq N\left\|e^{\alpha\left|x R^{-1}+\varphi(t) e_{1}\right|^{2}} H v\right\|_{L_{x}^{2} L_{t}^{2}}
\end{gathered}
$$

holds for any $\alpha \geq c R^{2}$ and $v \in C_{0}^{\infty}\left(\mathbb{R}^{n+1}\right)$ supported in the set

$$
\left\{(x, t):\left|x R^{-1}+\varphi(t) e_{1}\right| \geq 1\right\} .
$$

The proof of this lemma essentially follows that of Lemma 3.1 of [2]. However, the second term on the left-hand side of (3.1) does not appear in that lemma. For the sake of completeness, we provide the proof.

Proof. Set $f=e^{\alpha\left|x R^{-1}+\varphi(t) e_{1}\right|^{2}} v$. Direct computation yields

$$
e^{\alpha\left|x R^{-1}+\varphi(t) e_{1}\right|^{2}} H v=S_{\alpha} f-4 \alpha A_{\alpha} f
$$

where

$$
\begin{gathered}
S_{\alpha}=H+4 \alpha^{2} R^{-2}\left|x R^{-1}+\varphi(t) e_{1}\right|^{2} \\
A_{\alpha}=R^{-1}\left(x R^{-1}+\varphi(t) e_{1}\right) \cdot \nabla+d R^{-2} / 2+i \varphi^{\prime}\left(x_{1} R^{-1}+\varphi\right) / 2
\end{gathered}
$$

satisfying

$$
A_{\alpha}^{*}=-A_{\alpha}, \quad S_{\alpha}^{*}=S_{\alpha}
$$


Therefore

$$
\begin{gathered}
\left\|e^{\alpha\left|x R^{-1}+\varphi(t) e_{1}\right|^{2}} H v\right\|_{L_{x}^{2} L_{t}^{2}}^{2}=\left\langle S_{\alpha} f-4 \alpha A_{\alpha} f, S_{\alpha} f-4 \alpha A_{\alpha} f\right\rangle \\
\geq-4 \alpha\left\langle\left(S_{\alpha} A_{\alpha}-A_{\alpha} S_{\alpha}\right) f, f\right\rangle=-4 \alpha\left\langle\left[S_{\alpha}, A_{\alpha}\right] f, f\right\rangle .
\end{gathered}
$$

Since

$$
\begin{aligned}
& {\left[S_{\alpha}, A_{\alpha}\right]=2 R^{-2} \Delta-8 \alpha^{2} R^{-4}\left|x R^{-1}+\varphi e_{1}\right|^{2}} \\
& -\left[\left(x_{1} R^{-1}+\varphi\right) \varphi^{\prime \prime}+\left(\varphi^{\prime}\right)^{2}\right] / 2+2 i \varphi^{\prime} R^{-1} \partial_{x_{1}},
\end{aligned}
$$

the left-hand side of (3.2) is greater than or equal to

$$
\begin{gathered}
32 \alpha^{3} R^{-4} \int\left|x R^{-1}+\varphi(t) e_{1}\right|^{2}|f|^{2} d x d t+8 \alpha R^{-2} \int|\nabla f|^{2} d x d t \\
+2 \alpha \int\left[\left(x_{1} R^{-1}+\varphi\right) \varphi^{\prime \prime}+\left(\varphi^{\prime}\right)^{2}\right]|f|^{2} d x d t-8 \alpha i R^{-1} \int \varphi^{\prime} \partial_{x_{1}} f \bar{f} d x d t \\
\geq 32 \alpha^{3} R^{-4} \int\left|x R^{-1}+\varphi(t) e_{1}\right|^{2}|f|^{2} d x d t+8 \alpha R^{-2} \int|\nabla f|^{2} d x d t \\
-N \alpha \int\left(1+\left|x_{1} R^{-1}+\varphi\right|\right)|f|^{2} d x d t-N \alpha R^{-1} \int|\nabla f||f| d x d t \\
\geq 16 \alpha^{3} R^{-4} \int\left|x R^{-1}+\varphi(t) e_{1}\right|^{2}|f|^{2} d x d t+4 \alpha R^{-2} \int|\nabla f|^{2} d x d t,
\end{gathered}
$$

for $\alpha \geq c R^{2}$, with $N=N\left(\left\|\varphi^{\prime}\right\|_{L^{\infty}},\left\|\varphi^{\prime \prime}\right\|_{L^{\infty}}\right)$. Note that the last inequality is a consequence of the support property of $v$ and the Cauchy-Schwarz inequality. Using the inequality $|x-y|^{2}+|y|^{2} \geq \varepsilon|x|^{2}+\varepsilon|y|^{2}$, for some $\varepsilon=\varepsilon(d)>0$, the estimate (3.1) follows.

We are now ready to obtain the following lower bound.

Theorem 3.2. Let $u \in C\left([0,1]: H^{1}\right)$ be a solution of (1.3). If for some $\delta_{1}, \delta_{2}, L_{1}$, $L_{2}>0$ and $R_{1} \geq 1$

$$
\begin{gathered}
\int_{2 \delta_{2}}^{1-2 \delta_{2}} \int_{|x| \leq R_{1}}|u|^{2} d x d t \geq \delta_{1}, \\
\left\|V_{1}\right\|_{L_{t, x}^{\infty}}+\left\|V_{2}\right\|_{L_{t, x}^{\infty}} \leq L_{1}, \quad\left\|\left|p_{1}\right|\right\|_{L_{t, x}^{\infty}}+\left\|p_{2} \mid\right\|_{L_{t, x}^{\infty}} \leq L_{2},
\end{gathered}
$$

then there exist $R_{0} \geq R_{1}+1$ depending on $d, \delta_{1}, \delta_{2}, L_{1}$ and $A:=\|u\|_{L^{2}\left([0,1]: H^{1}\right)}$, and a constant $c_{1} \geq 1$ depending only on $d$ and $L_{2}$ such that for any $R \geq R_{0}$

$$
\delta(R):=\|u\|_{L^{2}\left([0,1]: H^{1}(\{R-1 \leq|x| \leq R\})\right)} \geq e^{-c_{1} R^{2}} .
$$

Proof. Following 2], for any $R>1$ we define smooth functions $\theta_{R}, \eta \in C_{0}^{\infty}\left(\mathbb{R}^{n}\right)$ and $\varphi \in C_{0}^{\infty}([0,1])$ satisfying

$$
\begin{aligned}
& \left\{\begin{array} { l l } 
{ \theta _ { R } = 1 , } & { \text { if } | x | \leq R - 1 , } \\
{ \theta _ { R } = 0 , } & { \text { if } | x | \geq R , }
\end{array} \quad \left\{\begin{array}{ll}
\eta=0, & \text { if }|x| \leq 1, \\
\eta=1, & \text { if }|x| \geq 2,
\end{array}\right.\right. \\
& 0 \leq \varphi \leq 3, \quad\left|\phi^{\prime}\right| \leq 4 \delta_{2}^{-1}, \quad \begin{cases}\varphi=0, & \text { in }\left[0, \delta_{2}\right] \cup\left[1-\delta_{2}, 1\right], \\
\varphi=3, & \text { if }\left[2 \delta_{2}, 1-2 \delta_{2}\right] .\end{cases}
\end{aligned}
$$

We apply Lemma 3.1 to the function

$$
v(x, t)=\theta_{R}(x) \eta\left(x R^{-1}+\varphi(t) e_{1}\right) u(x, t), \quad(x, t) \in \mathbb{R}^{d} \times[0,1],
$$


and observe that $v$ is supported in $\{|x| \leq R\} \times\left[\delta_{2}, 1-\delta_{2}\right]$ and satisfies the hypothesis of Lemma 3.1. Moreover, on $B_{R-1} \times\left[2 \delta_{2}, 1-2 \delta_{2}\right]$ we have $v=u$ and $\left|x R^{-1}+\varphi e_{1}\right| \geq$ 2. Thus,

$$
\left\|e^{\alpha\left|x R^{-1}+\varphi e_{1}\right|^{2}} v\right\|_{L_{x}^{2} L_{t}^{2}} \geq e^{4 \alpha}\|u\|_{L_{x}^{2}\left(\left\{|x| \leq R_{1}\right\}\right) L_{t}^{2}\left(\left[2 \delta_{2}, 1-2 \delta_{2}\right]\right)} \geq e^{4 \alpha} \delta_{1} .
$$

We compute

$$
\begin{gathered}
H_{V, p} v=\eta\left(x R^{-1}+\varphi e_{1}\right)\left(2 \nabla \theta_{R}(x) \cdot \nabla u+u \Delta \theta_{R}(x)-\left(u a_{1}+\bar{u} a_{2}\right) \cdot \nabla \theta_{R}(x)\right) \\
+\theta_{R}(x)\left[2 R^{-1} \nabla \eta\left(x R^{-1}+\varphi e_{1}\right) \cdot \nabla u+R^{-2} u \Delta \eta\left(x R^{-1}+\varphi e_{1}\right)\right. \\
\left.+i \varphi^{\prime} \partial_{x_{1}} \eta\left(x R^{-1}+\varphi e_{1}\right) u-\left(u a_{1}+\bar{u} a_{2}\right) \cdot R^{-1} \nabla \eta\left(x R^{-1}+\varphi e_{1}\right)\right] \\
\quad+R^{-1} \nabla \theta_{R} \cdot \nabla \eta\left(x R^{-1}+\varphi e_{1}\right) u .
\end{gathered}
$$

Notice that the first term on the right-hand side of (3.6) is supported in $B_{R}$ । $B_{R-1} \times[0,1]$ where $\left|x R^{-1}+\varphi e_{1}\right| \leq 4$, while the second and the third terms are supported in $\left\{(x, t): 1 \leq\left|x R^{-1}+\varphi e_{1}\right| \leq 2\right\}$. Therefore Lemma 3.1 with $\alpha=c R^{2}$ yields

$$
\begin{gathered}
N^{-1}\left(c^{3 / 2} R\left\|e^{\alpha\left|x R^{-1}+\varphi e_{1}\right|^{2}} v\right\|_{L_{x}^{2} L_{t}^{2}}+c^{1 / 2}\left\|e^{\alpha\left|x R^{-1}+\varphi e_{1}\right|^{2}}|\nabla v|\right\|_{L_{x}^{2} L_{t}^{2}}\right) \\
\leq L_{1}\left\|e^{\alpha\left|x R^{-1}+\varphi e_{1}\right|^{2}} v\right\|_{L_{x}^{2} L_{t}^{2}}+L_{2}\left\|e^{\alpha\left|x R^{-1}+\varphi e_{1}\right|^{2}}|\nabla v|\right\|_{L_{x}^{2} L_{t}^{2}} \\
+e^{16 \alpha} \delta(R)+e^{4 \alpha} A \delta_{2}^{-1} .
\end{gathered}
$$

Taking $R \geq R_{0}\left(L_{1}, d\right)$ and $c \geq c_{0}\left(L_{2}, d\right)$ sufficiently large, the first two terms on the right-hand side of (3.7) can be absorbed in the left-hand side. This combined with (3.5) yields

$$
N^{-1} c^{1 / 2} R \delta_{1} \leq e^{12 \alpha} \delta(R)+A \delta_{2}^{-1}
$$

To complete the prove of the theorem, it suffices to set $c_{1}=12 c$ and choose $R \geq$ $R_{0}\left(d, L_{1}, \delta_{1}, \delta_{2}, A\right)$.

\section{Proof of Theorems 1.1 and 1.3}

With the upper and lower bounds established in Sections 2 and 3, we are ready to prove Theorems 1.1 and 1.3 .

Proof of Theorem 1.1. Without loss of generality, we may assume that $u$ satisfies (3.3) for some $R_{1}, \delta_{1}$ and $\delta_{2}$. Indeed, if (3.3) does not hold for any $R_{1}, \delta_{1}$ and $\delta_{2}$, then $u \equiv 0$ on $\mathbb{R}^{d} \times[0,1]$, and there is nothing to prove. By Theorems 2.4 and 3.2 , we have for $R$ sufficiently large

$$
e^{-c_{1}\left(d, L_{2}\right)(R+1)^{2}} \leq N(d) e^{-a R^{2} / 36 d} .
$$

But this is impossible if $a \geq c_{0}:=37 c_{1} d$. Hence the theorem follows.

Proof of Theorem [1.3. By using Theorem 1.1 and considering the difference $u:=$ $u_{1}-u_{2}$ of two different solutions of (1.6), the proof of the theorem follows that of Corollary 1.2 of [6] almost word by word. Therefore, it will be omitted.

\section{ACKNOWLEDGMENTS}

The authors would like to express their gratitude to Carlos Kenig for bringing this problem to their attention and for many enlightening discussions. 


\section{REFERENCES}

[1] J. Bourgain, On the compactness of support of solutions of dispersive equations, Internat. Math. Res. Not. 9 (1997), 437-447. MR1443322 (98a:35113)

[2] L. Escauriaza, C.E. Kenig, G. Ponce, L. Vega, On unique continuation of solutions of Schrödinger equations, to appear in Comm. PDE.

[3] L. Escauriaza, C.E. Kenig, G. Ponce, L. Vega, On uniqueness properties of solutions of the $k$-generalized $\mathrm{KdV}$ equations, preprint.

[4] C.E. Kenig, G. Ponce, L. Vega, Smoothing effects and local existence theory for the generalized nonlinear Schrödinger equations.Invent. Math. 134 (1998), no. 3, 489-545. MR1660933 (99k:35166)

[5] A.D. Ionescu, C.E. Kenig, $L^{p}$ Carleman inequalities and uniqueness of solutions of nonlinear Schrödinger equations, Acta Math. 193 (2004), no. 2, 193-239. MR2134866 (2005m:35283)

[6] A.D. Ionescu, C.E. Kenig, Uniqueness properties of solutions of Schrödinger equations, J. Funct. Anal. 232 (2006), 90-136. MR2200168

[7] C.E. Kenig, G. Ponce, L. Vega, On unique continuation for nonlinear Schrödinger equations, Comm. Pure Appl. Math. 56 (2003), pp. 1247-1262. MR1980854 (2004g:35195)

[8] C.E. Kenig, G. Ponce, L. Vega, The Cauchy problem for quasi-linear Schrödinger equations, Invent. Math. 158 (2004), 343-388. MR2096797 (2005f:35283)

School of Mathematics, Institute for Advanced Study, 1 Einstein Drive, Princeton, NEW JERSEY 08540

E-mail address: hjdong@ias.edu

Department of Mathematics, University of Chicago, 5734 S. University Avenue, Chicago, Illinois 60637

E-mail address: wolf@math.uchicago.edu 